\title{
Increasing the efficiency of high-order harmonic generation using counterpropagating laser pulses
}

\author{
I. P. Prokopovich,* A. A. Khrutchinsky, and D. Yu. Churmakov \\ Institute of Nuclear Problems, Belarusian State University, Bobruiskaya 11, Minsk 220050, Belarus \\ J. Peatross \\ Department of Physics and Astronomy, Brigham Young University, Provo, Utah 84602 \\ A. A. Apolonsky \\ Institute of Automation and Electrometry SB RAS, Novosibirsk, Russia
}

(Received 18 April 2001; published 10 May 2002)

\begin{abstract}
We present results of computer simulations showing dramatic increases in the production and intensity of high-order harmonics (in the cutoff region) produced by 20 -fs pulse with counterpropagating pulse in rare gases. Using two different models for the gas medium (i.e., classical anharmonic oscillators representing Ne, Ar, $\mathrm{Kr}$ and quantum oscillators representing $\mathrm{Ne}^{+}, \mathrm{Ar}^{+}, \mathrm{Kr}^{+}$), we demonstrate an efficiency increase of four and six orders of magnitude for the respective models. This suggests a notable improvement over other methods.

DOI: 10.1103/PhysRevA.65.053823

PACS number(s): 42.65.Ky, 42.50.Gy, 42.50.Hz
\end{abstract}

\section{INTRODUCTION}

High-order harmonic generation (HHG) by a strong laser field in gaseous media (see, for example, Refs. [1-4]) is an attractive source of coherent extreme ultraviolet and x-ray radiation. However, the efficiency of converting the energy of the driving laser pulse into high harmonics is at best $10^{-8}$ in the $20-\mathrm{nm}$ range, and below $10^{-10}$ in the $10-\mathrm{nm}$ range $[5,6]$. In an effort to increase the efficiency, HHG has been studied in a variety of media under a wide range of driving laser parameters (see, for example, Refs. [1-9], and references therein). Significant improvements to the efficiency of HHG can be made by decreasing the laser-pulse duration [2-4] and by designing laser-pulse temporal profiles to enhance the atomic response [9]. Nevertheless, the efficiency of converting laser field energy into high harmonics is limited in large measure by phase mismatches [5,10-13].

Tamaki et al. [14] demonstrated a 40-times increase in the conversion efficiency of harmonic orders near the 49th (in the cutoff region) by exploiting phase matching in a selfguided beam. Recently, Tempea et al. [15] proposed a mechanism of nonadiabatic self-phase matching to enhance HHG above $1 \mathrm{keV}$ by as much as four orders of magnitude. The nonadiabatic self-phase-matching scheme is expected to work predominantly for wavelengths in x-ray regime produced with very short laser-pulse durations (5 fs) at relatively high peak intensities $\left(I_{0}=1.5 \times 10^{16} \mathrm{~W} / \mathrm{cm}^{2}\right)[15]$.

Theoretical analysis has demonstrated that quasiphase matching [16] based on a modulated density of neutral and ionized gases $[10,13]$ has the potential to improve the conversion efficiency of HHG by about two orders of magnitude [10]. This can compensate for geometrical phase mismatches. However, this cannot deal with index-related phase mismatches, assuming them to be inseparably associated with the density modulation. Furthermore, the manipulation

\footnotetext{
*Email address: prok@inp.minsk.by
}

of the atomic density to create sufficiently precise structures in a gas density distribution poses some technical challenges.

An alternative approach to quasiphase matching of HHG was proposed in Ref. [11]. Rather than attempting to modulate spatially either the density of the atomic medium or the density of free electrons, counterpropagating light pulses (CPP) can be used to disrupt the harmonic production in selected zones with undesirable phase. The remaining zones then constructively interfere, and overall harmonic production increases. The CPP-based quasiphase matching of HHG was studied [11] for a simple phenomenological model of HHG and relatively weak counterpropagating light (one hundredth the intensity). No attempt was made to investigate the nonlinear dynamics of the medium polarization and its effect on propagation and reshaping of the driving pulse in the field of the CPP.

In this paper we present numerical investigations of the nonlinear dynamics (including the HHG process) of colliding laser pulses with equal peak intensities. The numerical data that is reported represent exact solutions to self-consistent equations, namely, Maxwell's equations associated with the laser fields and model equations representing the gaseous media. The latter consists of ensembles of (1) classical anharmonic oscillators [17] representing the behavior of neutral atoms and (2) two-level quantum oscillators associated with the fine structure of the ground state of their ions [18]. The time-dependent Schrödinger equation has often been employed to study HHG $[19,20]$ (requiring significant computational resources) where the Hamiltonian is applied to a model atom under the single-active electron approximation. However, a number of simplified models such as the classical anharmonic oscillator [7,17], two-level systems associated with direct transitions [21], or two-level systems associated with Raman transitions [18], give results that are qualitatively similar to those of the more exact calculations $[19,20]$. It was recently shown [22] that an ionizing two-level system, in which the ionized population is treated as classical particles moving inside the electric field, gives results that 
are in reasonable agreement with results obtained from integrating the Schrödinger equation $[19,20]$.

The results obtained by solving the time-dependent Schrödinger equation and the time-dependent Hartree-Fock equations [23] at intensities significantly above $10^{14} \mathrm{~W} / \mathrm{cm}^{2}$ showed that ions can emit harmonics with much higher order than neutrals. The theoretical model for HHG based on stimulated electronic Raman scattering (SERS) associated with the fine structure of singly ionized noble gases was studied [18] and shown to be in reasonable agreement with known attributes of HHG. In any case, the results presented in this paper do not depend sensitively on the model of the medium. We have studied HHG with single pulses as well as with CPP, using the two different models mentioned above. We show that both models give qualitatively similar results in the presence of CPP. The similarities include an increase in the number of harmonics (i.e., the intensity of the highest harmonics increases by several orders of magnitude) accompanied by a decrease in intensities of lower harmonic orders.

\section{CLASSICAL ANHARMONIC OSCILLATORS AND HHG WITH CPP}

\section{A. Maxwell's equations in one-dimension representing the laser field}

We begin with Maxwell's equations in one-dimension (1D), which describe the laser field polarized linearly in the $x$ direction and propagating in the $z$ direction. Written in Gaussian units, the equations take the form

$$
\frac{1}{c} \frac{\partial E_{x}}{\partial t}=-\frac{\partial H_{y}}{\partial z}-\frac{4 \pi}{c} \frac{\partial P}{\partial t} ; \quad \frac{1}{c} \frac{\partial H_{y}}{\partial t}=-\frac{\partial E_{x}}{\partial z},
$$

where, for the first model for the medium (noble gas atoms represented as classical anharmonic oscillators), the polarization is $P=-N_{\text {atom }} e q, N_{\text {atom }}$ is the density of atoms, $-e$ is the electronic charge, and $q$ is the displacement from equilibrium of an electron within an individual atom. The anharmonic oscillator equation corresponding to a centrosymmetric medium $[7,17,24]$ is

$$
\ddot{q}+\Gamma \dot{q}+\omega_{a o}^{2} q=-e m_{e} E_{x}(t, z)-\beta q^{3},
$$

where $\Gamma$ is a damping term, $\omega_{a o}$ is a characteristic frequency, and $\beta$ is the parameter of anharmonicity. For the model of HHG based on Eq. (2) with a laser pulse having frequency $\omega$, the ratio $\omega / \omega_{a o}$ is considered to be of the order of 0.1 [17], corresponding to the typical ratio between the photon energy of a terawatt laser (usually Ti:sapphire), and the ionization potential of a noble gas. It should be noted, that in this paper we avoid relativistic intensities $\left(10^{18} \mathrm{~W} / \mathrm{cm}^{2}\right)$, and thus the influence of the magnetic field on HHG may be neglected.

\section{B. Results of the anharmonic oscillator model for the medium}

We integrate Eq. (2) using standard fourth-order RungeKutta algorithms. The spectra of HHG that we obtained are similar to the results of the analogous model under similar conditions [17]. However, the influence of the laser-pulse

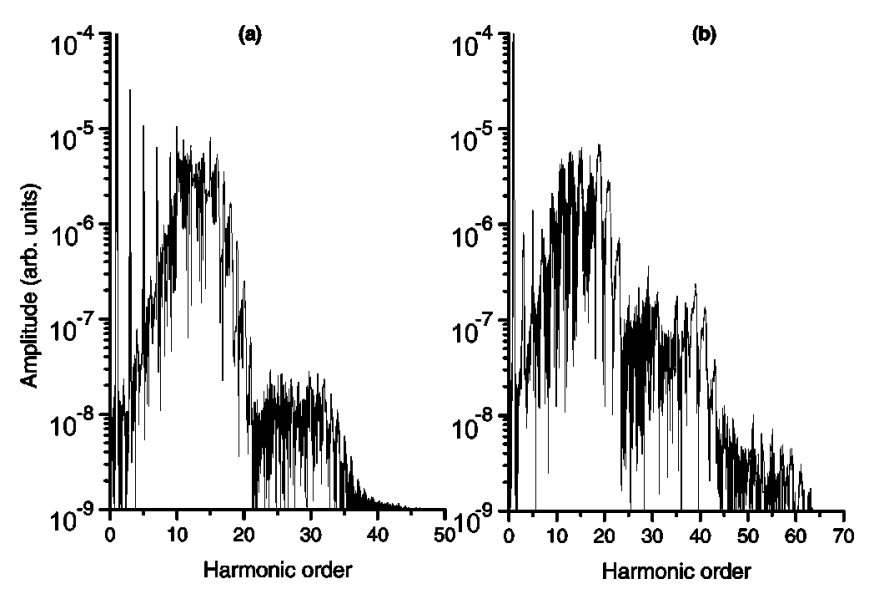

FIG. 1. Amplitude spectra vs $\omega / \omega_{0}$ of the 20-fs FWHM Gaussian pulse, $\lambda=800$-nm fundamental, of peak intensity $1.6 \times 10^{14}$ $\mathrm{W} / \mathrm{cm}^{2}$, passed $6 \mu \mathrm{m}$ in medium Duffing anharmonic oscillators with $N_{\text {atom }}=4 \times 10^{18}$ atoms $/ \mathrm{cm}^{3}, \omega_{0} / \omega_{a o}=0.1$, and the parameter of the anharmonicity $\beta=2 \times 10^{35}$ ( $\left.\AA \mathrm{s}\right)^{-2}$ typical for $\mathrm{Kr}$ (a) without using the 160-fs FWHM Gaussian counterpropagating pulse and (b) with using one.

propagation on the HHG process and its relation to collective phase-matching effects was not studied in Ref. [17]. The numerical integration of 1D Maxwell's equations (1) was performed using well-known algorithms [25].

We have numerically solved the self-consistent coupled Eqs. (1) and (2) for different durations and intensities of laser pulses. We found that the model shows a rise in the efficiency of HHG with decreasing laser-pulse duration. These results are in qualitative agreement with experimentally observed properties of HHG, as well as with the known behavior of other models (see, for example, Refs. $[17,26])$. Because one of the ways to increase the efficiency of HHG is to decrease the laser-pulse duration [2-4], we use as an initial pulse envelope a Gaussian with duration $\tau_{0}=20$-fs full width at half maximum (FWHM). Such 20-fs pulses from a Ti:sapphire laser (wavelength $\lambda \sim 800 \mathrm{~nm}$ ), contain only eight optical cycles FWHM.

Figure 1(a) shows the high harmonic spectrum associated with the 20 -fs pulse passing through a $6-\mu \mathrm{m}$ medium with $N_{\text {atom }}=4 \times 10^{18}$ atoms $/ \mathrm{cm}^{3}$. The initial intensity of the pulse was chosen to be $I_{0}=1.6 \times 10^{14} \mathrm{~W} / \mathrm{cm}^{2}$. In Eq. (2) we used as the parameter of anharmonicity $\beta=2 \times 10^{35}(\AA \mathrm{s})^{-2}$, which has been obtained for $\mathrm{Kr}$ based on experimental estimates [7]. The ratio between the laser frequency and the natural frequency of the oscillators was chosen to be $\omega_{0} / \omega_{a o}=0.1$, and the damping parameter was set to zero $(\Gamma=0)$. The spectrum in Fig. 1(a) exhibits the characteristic cutoff near the 35th harmonic. So-called "hyper-Raman" peaks are observed to the sides of harmonics with orders higher than seven. The hyper-Raman peaks in the harmonic spectra, exhibited in both the classical anharmonic oscillator and quantum oscillator case, were discussed in Ref. [17] (and references therein) and shown to be connected with dynamical coherence effects. Figure 1(b) shows the high harmonic spectrum generated by the same pulse when it passes through the medium in the presence of a 160 -fs counterpropagating pulse with the same peak intensity. A comparison of Figs. 
1(a) and 1(b) reveals that the CPP alters radically the spectrum of the 20-fs pulse: (1) The maximum harmonic photon energy increases by more than 60\% (the 39th harmonic is the maximum order when the CPP is not used [Fig. 1(a)], and the 65th harmonic is the maximum order when the CPP is used [Fig. 1(b)]). (2) The amplitude of higher harmonics increases (for instance, the amplitudes of the 39th and 37th harmonics are increased by two orders of magnitude, that is, the intensities of these harmonics are increased by four orders of magnitudes). (3) The amplitude of the lower harmonics decreases. We performed computer simulations for different durations of the CPP, although Fig. 1(b) shows only the result with a 160 -fs CPP. A longer CPP is able to interact with the forward-traveling pulse over a comparatively longer distance. Our simulations show that if the duration of the CPP is decreased, HHG in the direction of the CPP increases while the efficiency of HHG from the 20-fs pulse falls. The latter is easy to understand since a decrease in the duration of the CPP leads to increased HHG from that pulse (traveling opposite to the propagating 20 -fs pulse). At the same time, the interaction length for both pulses is shortened, decreasing the efficiency of converting the 20-fs field into high harmonics. One might expect a further rise in the efficiency of HHG from the 20-fs pulse by increasing the CPP duration indefinitely. However, calculations, which take into account ionization and the influence of the resulting plasma on pulse propagation (see Sec. IV), indicates that coherence lengths for many of high-order harmonics are less than the interaction length set up by a $160-$ fs CPP.

\section{HHG AT THE FINE STRUCTURE OF SINGLY IONIZED NOBLE GASES AND USING CPP}

It is well known that at intensities of the order of $I_{0}$ $\sim 10^{14} \mathrm{~W} / \mathrm{cm}^{2}$ and higher (with wavelength $\sim 800 \mathrm{~nm}$ ), ionization of noble gases occurs on every half optical cycle of the field via a tunneling process. For example, at an intensity of $3 \times 10^{14} \mathrm{~W} / \mathrm{cm}^{2}$, approximately half of the argon atoms present are ionized by the peak of the 27 -fs pulse [26]. In addition, Krause et al. [23] suggested that at intensities higher than $10^{14} \mathrm{~W} / \mathrm{cm}^{2}$ ions may contribute significantly to the harmonic spectra.

\section{A. Fine structure of singly ionized noble gases as a two-level system for SERS and HHG}

Next we turn to a model for HHG, which is based on SERS [18] associated with the fine structure of singly ionized noble gases. SERS can be realized on transitions between fine-structure ${ }^{2} P_{3 / 2^{-}}{ }^{2} P_{1 / 2}$ levels of the $\mathrm{Ne}^{+}, \mathrm{Ar}^{+}$, $\mathrm{Kr}^{+}, \mathrm{Xe}^{+}$ions. For example, in $\mathrm{Ne}^{+}$the ground state ${ }^{2} P_{3 / 2}$ and the neighboring excited state ${ }^{2} P_{1 / 2}$ form a two-level system, where the frequency transition is $\Omega /(2 \pi c)$ $=780.4 \mathrm{~cm}^{-1}$. The transitions are forbidden by parity in the electrodipole approximation for a one-quantum process but permitted for a two-quantum process such as SERS. All other levels are far removed from $\Delta>217047 \mathrm{~cm}^{-1}$ [27]. A similar situation exists for the ions of the other noble gases. Seeing that a 20 -fs pulse has a spectral bandwidth in the range of $1668 \mathrm{~cm}^{-1}$ the transition frequency $\Omega$ is easily contained within the spectrum of the pulse, i.e., its frequency range contains a continuous set of components that are comparable in amplitude and satisfy the condition of the Raman resonance. This allows effective stimulated Raman selfscattering (SRSS) [18]. The frequency $\Omega$ is much less than the central frequency $\omega_{o}$ of the initial laser-pulse field $\mathcal{E}$. The average value of polarizability is $\langle\alpha\rangle=S p(\alpha \rho)$ and the difference of the level populations is $w=\rho_{22}-\rho_{11}$, where $\alpha$ is the operator of polarizability of the medium, and $\rho$ is the density matrix, which are described by the equations $[18,28]$

$$
\begin{gathered}
\frac{\partial^{2}\langle\alpha\rangle}{\partial t^{2}}+\frac{2}{T_{2}} \frac{\partial\langle\alpha\rangle}{\partial t}+\Omega^{2}\langle\alpha\rangle=-\left|\alpha_{12}\right|^{2} \frac{\Omega}{\hbar} \mathcal{E}^{2} w, \\
\frac{\partial w}{\partial t}+\frac{\left(w-w_{o}\right)}{T_{1}}=\frac{1}{\hbar \Omega} \mathcal{E}^{2}\left(\frac{\partial\langle\alpha\rangle}{\partial t}+\frac{\langle\alpha\rangle}{T_{2}}\right),
\end{gathered}
$$

where $\left|\alpha_{12}\right|$ is a value of the matrix element of the polarizability corresponding to the Raman transition with frequency $\Omega$. The parameter $T_{2}$ is the dephasing time or the time of Raman coherence, and $T_{1}$ is the time of population relaxation. Since we will consider coherent interactions only ( $\tau_{0}$ $\left.\ll T_{2}, T_{1}\right)$, we can neglect the terms related to the relaxation times and in Eqs. (3) and (4). The nonlinear dipole moment related to SRSS is $d=\alpha \mathcal{E}$. In this case, the nonlinear contribution to the polarization of the medium is given by $P$ $=N_{\text {ion }}\langle\alpha\rangle \mathcal{E}$, where $N_{\text {ion }}$ is the number density of noble gas ions present. The 1D Maxwell's equations (1) together with the expressions for the polarization (3) and (4) (describing the medium as an ensemble of the two-level oscillators under two-quantum Raman-type transitions) forms a system of self-consistent equations for the second model investigated in this paper.

\section{B. Results for the fine-structure model of ionized media}

The results of our computer simulation for this model are shown in Fig. 2. The initial pulse and CPP have the parameters identical to those used for the anharmonic oscillator model used above. The 20 -fs pulse passed through the same $6-\mu \mathrm{m}$ distance in medium with $N_{\text {ion }}=3 \times 10^{18} \mathrm{ions} / \mathrm{cm}^{3}$. A lower limit for the matrix element $\left|\alpha_{12}\right|$ of the polarizability can be estimated from experimental data on multiphoton ionization in noble gases. For example, in experiments [29] with initial pulses $\tau_{0}=1$ ps and intensities $I_{o} \sim 10^{13} \mathrm{~W} / \mathrm{cm}^{2}$ an effective change in the population of the states ${ }^{2} P_{3 / 2}$ and ${ }^{2} P_{1 / 2}$ in $\mathrm{Kr}^{+}$ions was observed. This means that the corresponding two-photon Rabi frequency can be estimated as $\Omega_{2 p h R} \gg 1 / \tau_{0}$. If we take into consideration that $\left|\alpha_{12}\right| \sim \Omega_{2 p h R} \hbar\left(c / I_{o}\right)$ [21] for $\Omega_{2 p h R}=3 \times 10^{12} \mathrm{~s}^{-1}$ and $I_{o}$ $\sim 10^{13} \mathrm{~W} / \mathrm{cm}^{2}$ [29] we obtain $\left|\alpha_{12}\right| \sim 10^{-24} \mathrm{~cm}^{3}$. We used this value $\left|\alpha_{12}\right|$ in the calculations illustrated in Figs. 2 and 3. Qualitatively, the results shown in Fig. 2 are similar to those exhibited by the anharmonic oscillator model, but with a somewhat more dramatic effect: (1) we see an increase in the maximum harmonic order of more than two times (the 39th harmonic is the maximum when the CPP was not used [Fig. $2(\mathrm{a})]$, and the plateau cutoff is in the region of the 85th harmonic when the CPP was used [Fig. 2(b)]); (2) the am- 


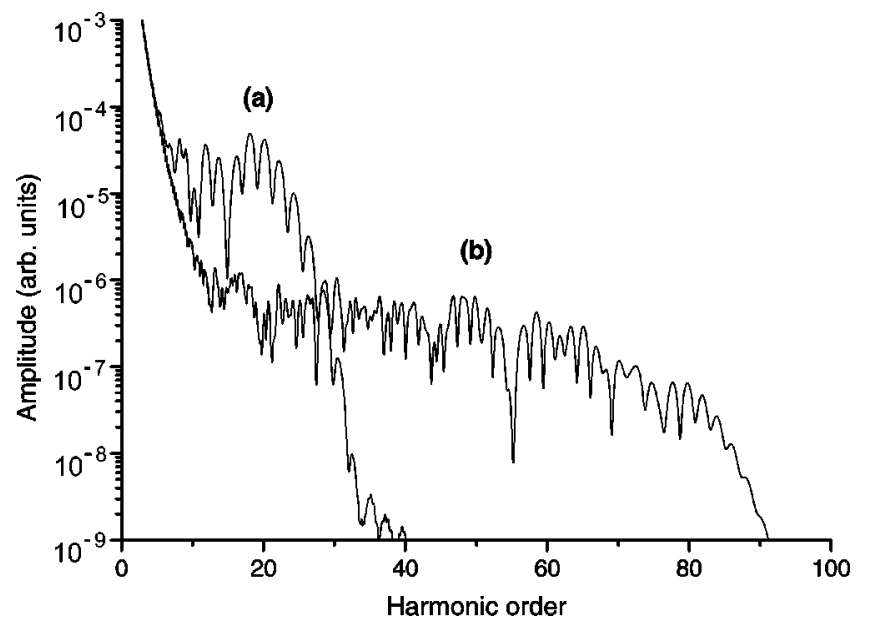

FIG. 2. Amplitude spectra vs $\omega / \omega_{0}$ of the $20 \mathrm{fs}$ pulse, with the same parameters as in Fig. 1, passed under SERS at the fine structure, $\Omega / \omega_{0}=0.1$, of ions with $N_{\text {ion }}=3 \times 10^{18} \mathrm{ions} / \mathrm{cm}^{3}$, and $\left|\alpha_{12}\right|$ $=10^{-24} \mathrm{~cm}^{3}$ (a) without using the 160 -fs counterpropagating pulse and (b) with using one.

plitude of higher harmonics (for instance, the 39th and 37th) increases by about three orders of magnitude, that is, the intensities of these harmonics increase by about six orders of magnitude; (3) the amplitude of the lower harmonics (up to 23rd) decreases. It is seen that the efficiency of HHG for the quantum oscillators (see Fig. 2) is significantly higher than that for the anharmonic oscillator model (see Fig. 1). This is easy to understand in view of the fact that in the classical anharmonic oscillator [Eq. (2)] the third-order nonlinearity is taken into account only, and in the Eqs. (3) and (4) for the two-level system all the higher-order nonlinearities are retained automatically [the difference of the level populations $w$ is the intensity-dependent function described in Eq. (4)].

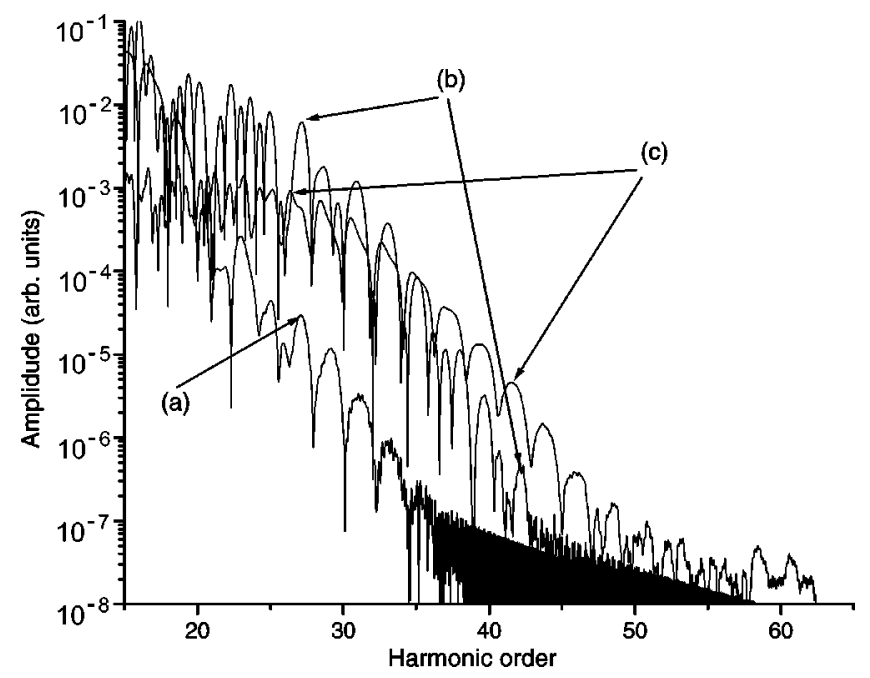

FIG. 3. Amplitude spectra vs $\omega / \omega_{0}$ of the 20 -fs pulse (a) with peak intensity $3.2 \times 10^{14} \mathrm{~W} / \mathrm{cm}^{2}$ without CPP, (b) with peak intensity $6.4 \times 10^{14} \mathrm{~W} / \mathrm{cm}^{2}$ without CPP, (c) with peak intensity 1.6 $\times 10^{14} \mathrm{~W} / \mathrm{cm}^{2}$ after interreaction with the same CPP. All parameters of the medium are the same as in Fig. 2.

\section{HHG using CPP vs HHG using single pulses}

To demonstrate that these effects cannot be obtained by simply raising of the intensity of the 20 -fs pulse taken alone, we performed computer simulations, using the model for the quantum oscillators, for a single 20 -fs pulse with initial intensity twice and four times as large as that in Fig. 2. This exceeds, on average, the intensity of the combined pulses, the forward and the counterpropagating pulses used to generate Fig. 2. All parameters regarding the medium are the same ones as used for Fig. 2. Figures 3(a) and 3(b) show the spectra (in the cutoff region) generated by the single $20-\mathrm{fs}$ pulse with different intensities passing through the same medium as in Fig. 2. Figure 3(c) shows the result of a lowerintensity pulse that encounters an identical CPP. As seen in Figs. 3(b) and 3(c), the amplitude of the lower-order harmonics (up to 33rd) are higher from a pulse with quadrupled intensity $I_{0}=6.4 \times 10^{14} \mathrm{~W} / \mathrm{cm}^{2}$, but, at the same time, the amplitude of the higher-order harmonics (beyond 33rd) are lower in amplitude than those from the pulse and identical CPP pair (each at $I_{0}=1.6 \times 10^{14} \mathrm{~W} / \mathrm{cm}^{2}$ ). For instance, the amplitude of the 43rd harmonic [in the case of Fig. 3(c) as opposed to Fig. 3(b)] is higher by an order of magnitude. That is, the intensity of that harmonic is higher by two orders of magnitude. In addition, as is clear from the Fig. 3, the use of the CPP allows us to reach higher photon energies [the 43rd harmonic is the maximum order in the case (b), and the 61 st harmonic is the maximum order in the case (c)].

\section{Plasma and coherent lengths}

Finally, we have calculated the effect of plasma on the coherence length of the interaction. We used as an estimate for the ion density $3 \times 10^{18}$ ions $/ \mathrm{cm}^{3}$. Taking the density of free electrons to be the same, the electron plasma frequency is $\omega_{p}=9.75 \times 10^{13} \mathrm{~s}^{-1}$. In this case, for the 39th harmonic $\left(\lambda_{39} \sim 20.5 \mathrm{~nm}\right)$ the coherence length $L_{e}=7.6 \mu \mathrm{m}$, and for the 61st harmonic $\left(\lambda_{61} \sim 13 \mathrm{~nm}\right)$ the coherence length $L_{e}$ $\sim 5 \mu \mathrm{m}\left(L_{c}=2 /\left(j k_{1}-k_{j}\right)\right.$. The wave number of the jth harmonic is given by $\left.k_{j}=\left[1-\left(\omega_{p} / \omega_{j}\right)^{2}\right]^{1 / 2} \omega_{j} / c\right)$. These coherence lengths are in the neighborhood of the propagation distance of $6 \mu \mathrm{m}$ used to generate Figs. 1, 2, and 3 for the 20-fs pulse.

\section{DISCUSSION AND CONCLUSIONS}

We have shown, using two different models of mediaclassical anharmonic oscillators (for $\mathrm{Ne}, \mathrm{Ar}, \mathrm{Kr}$ ) as well as quantum oscillators (for $\mathrm{Ne}^{+}, \mathrm{Ar}^{+}, \mathrm{Kr}^{+}$) - that the use of counterpropagating pulses leads to an increase in the generating efficiency of the highest harmonics by as much as four orders of magnitude. This also permits much higher photon energies. The CPP causes an increase in amplitude of higher harmonics and a simultaneous decrease in amplitudes of lower harmonics. This attribute is common to both the classical and quantum models. A single pulse with higher initial intensity (e.g., four times to accomplish the same peak intensity and higher one, on average) is unable to match the effect. The effect occurs when the medium is relatively short (i.e., $6 \mu \mathrm{m}$ ), and manifests itself most strongly when the 
quantum oscillator model is used. This is not surprising since that model retains automatically all of the higher-order nonlinearities. The results show a dependence on the duration and interaction length of the pulses.

The interference between the driving and counterpropagating pulses induces a united wave field. This superposition field has a steeper field gradient than the 20 -fs pulse by itself. The field of the 20-fs driving pulse, which contains only eight optical cycles FWHM with an initial Gaussian envelope, is reshaping more strongly (at its front and tail) during the interaction with the counterpropagating pulse, than if in case of longer driving pulse. Recently, Bartels et al. [9] demonstrated experimentally, using $\sim 20$-fs $(\lambda \sim 800 \mathrm{~nm})$ pulses with peak intensity $\sim 2 \times 10^{14} \mathrm{~W} / \mathrm{cm}^{2}$, that a very slight temporal reshaping of the driving pulse can dramatically influence the harmonic radiation, increasing the intensities of the higher harmonics [25th,27th (by an order of magnitude), 29th,31st] while suppressing the intensities of the lower harmonics. They found this effect to be very general, occurring over a wide range of parameters and in different noble gases. Their work illustrates significant differences between loworder nonlinear processes (second-, third-harmonic generation) and high-order processes such as HHG, which are inherently nonperturbative. No CPP was used in their work.

Counterpropagating light was used in recent experiments with a 30-fs driving pulse [30], where it was shown that a relatively weak (60 times less intense) 1-ps counterpropagating pulses can disrupt or boost, high-harmonics generation by as much as two orders of magnitude, depending on a position of gas jet relative to the laser focus. The $300-\mu \mathrm{m}$-diameter gas nozzle was positioned at the collision point of the two pulses. The authors interpreted the results in framework of a simple model of quasiphase matching $[11,31]$. In contrast, the conditions studied in the present work are quite different from the conditions used in Ref. [30]. The observed effects are also qualitatively different. The effect studied here occurs when the medium is relatively short $\sim 6 \mu \mathrm{m}$. Using the results from Ref. [12] and taking into account the parameters used in this paper, one can see that phase mismatches should not be important, including the geometrical mismatches and mismatches due to the dispersion of neutral atoms and free electrons (as noted above regarding plasma and coherent lengths). For instance, for the 61st harmonic, the coherence length relating to geometrical mismatches is $L_{\text {geo }} \sim 1 \mathrm{~mm}$. Also, our numerical experiments indicate that the enhancement effect strongly diminishes if the propagation distance of the driving pulse in medium is more than the interaction length $\left(L_{i n t}=24 \mu \mathrm{m}\right)$ of the driving (20-fs) and counterpropagating (160-fs) pulses. Our investigations show that when the intensity of the counterpropagating pulse $I_{c p p}$ decreases, the effect falls off and disappears altogether by the time $I_{c p p}$ is a factor of 10 less than the intensity of the driving pulse. By comparison, for the work Ref. [30] the counterpropagating intensity $I_{c p p}$ was even much less (60 times less).

The initial parameters of the driving pulse in our work are close to those used by Bartels' et al. [9]. Moreover, our work exhibits the redirection of energy into higher harmonics, in qualitative agreement with their experimental results [9]. Nevertheless, the results differ significantly in that their laser-atom interaction was modified through a pulse-shaping unique technique designed to optimize $\mathrm{HHG}$, whereas we modified the laser-atom interaction using counterpropagating pulse. Our numerical experiments show that a counterpropagating pulse (with the intensity that equal to the driving pulse) permits the redirection of energy into the higher harmonics. This takes place in a relatively short propagation distance (about the space length (at FWHM) of driving pulse).

The results presented here depend strongly on the shape of electromagnetic field and on the propagation distance, indicating that the effect exhibits properties of a coherent interaction. These properties and their physical interpretation are understood in terms of the self-consistent solution describing the interaction between the ensemble of atoms and the electromagnetic field, taking in account propagation. This is fundamentally different than a simple sum of the emission from individual atoms stimulated by a prescribed field. The quantitative properties of the effect are obtained only by means of a proper quantum description of the medium, taking into account all essential nonlinearities.

\section{ACKNOWLEDGMENTS}

This research was supported in part by INTAS, Projects Nos. 97-1058 and 97-2018, as well as by the Belarus Basic Research Foundation.
[1] A. McPherson et al. J. Opt. Soc. Am. B 4, 595 (1989); A.L.' Huillier et al. Phys. Rev. Lett. 70, 774 (1993); J.J. Macklin et al., ibid. 70, 766 (1993); M.D. Perry and G. Mourou, Science 264, 917 (1994).

[2] J. Zhou, J. Peatross, M.M. Murnane, and H.C. Kapteyn, Phys. Rev. Lett. 76, 752 (1996).

[3] Z. Chang, A. Rundquist, H. Wang, M.M. Murnane, and H.C. Kapteyn, Phys. Rev. Lett. 79, 2967 (1997).

[4] M. Schnurer et al., Phys. Rev. Lett. 80, 3236 (1998).

[5] T. Ditmire, K. Kulander, J.K. Crane, H. Nguyen, and M.D. Perry, J. Opt. Soc. Am. B 13, 406 (1996).

[6] T. Ditmire et al., Phys. Rev. A 51, R902 (1995); G. Sommerer et al., Laser Phys. 9, 1 (1998); Opt. Commun. 146, 347 (1998).
[7] J. Peatross and D.D. Meyerhofer, Phys. Rev. A 52, 3976 (1995).

[8] C. Lynga, A.L.' Huillier, and C.-G. Wahlstrom, J. Phys. B 29, 3293 (1996).

[9] B. Kohler et al., Phys. Rev. Lett. 74, 3360 (1995); R. Baitels et al., Nature (London) 406, 164 (2000).

[10] P.L. Shkolnikov, A.E. Kaplan, and A. Lago, J. Opt. Soc. Am. B 13, 412 (1996).

[11] J. Peatross, S. Voronov, and I. Prokopovich, Opt. Express 1, 108 (1997).

[12] C. Delfin et al., J. Phys. B 32, 5397 (1999).

[13] T. Brabec and F. Krausz, Rev. Mod. Phys. 72, 545 (2000).

[14] Y. Tamaki et al., Phys. Rev. Lett. 82, 1422 (1999). 
[15] G. Tempea, M. Geissler, M. Schnurer, and T. Brabec, Phys. Rev. Lett. 84, 4329 (2000).

[16] J.A. Armstrong, N. Blombergen, J. Ducuing, and P.S. Pershan, Phys. Rev. 327, 1918 (1962).

[17] Ph. Balcou, A.L.' Huillier, and D. Escande, Phys. Rev. A 53, 3456 (1996).

[18] I.P. Prokopovich and A.A. Khrutchinsky, Laser Phys. 6, 41 (1996).

[19] J.H. Eberly, Q. Su, and J. Vavanaingn, J. Opt. Soc. Am. B 6, 1289 (1989); Q. Su and J.H. Eberly, Phys. Rev. A 44, 5997 (1991).

[20] K.C. Kulander and B.W. Shore, Phys. Rev. Lett. 62, 524 (1989).

[21] B. Sundaram et al., Phys. Rev. A 41, 6571 (1990); L. Plaja et al., J. Opt. Soc. Am. B 9, 2210 (1992); A.E. Kaplan and P.L. Shkolnikov, Phys. Rev. A 49, 1275 (1994).

[22] E.C. Jarque and L. Plaja, J. Phys. B 31, 1687 (1998).

[23] J.L. Krause, K.J. Schafer, and K.C. Kulander, Phys. Rev. Lett.
68, 3535 (1992); H. Xu, X. Tang, and P. Lambropoulos, Phys. Rev. A 46, R2225 (1992).

[24] R.W. Boyd, Nonlinear Optics (Academic Press, San Diego, 1992).

[25] C.K. Birdsall and A.B. Langdon, Plasma Physics, via Computer Simulation (McGraw-Hill, New York, 1985).

[26] K.J. Schafer and K.C. Kulander, Phys. Rev. Lett. 78, 638 (1997).

[27] A.A. Radzig and B.M. Smirnov, Reference Data on Atoms, Molecules and Ions (Springer-Verlag, Berlin, 1985).

[28] R.H. Pantell and H.E. Puthoff, Fundamentals of Quantum Electronics (Wiley, New York, 1969).

[29] M.D. Perry, O.L. Landen, and A. Szöke, J. Opt. Soc. Am. B 6, 344 (1989).

[30] S.L. Voronov et al., Phys. Rev. Lett. 87, 133902 (2001).

[31] J. Peatross, J. Chaloupka, and D.D. Meyerhofer, Opt. Lett. 19, 942 (1994) 